\title{
Solvability for fractional order boundary value problems at resonance
}

\author{
Zhigang $\mathrm{Hu}^{*}$ and Wenbin Liu
}

\author{
* Correspondence: xzhzgya@126. \\ com \\ Department of Mathematics, China \\ University of Mining and \\ Technology, Xuzhou 221008 \\ People's Republic of China
}

\section{Abstract}

In this paper, by using the coincidence degree theory, we consider the following boundary value problem for fractional differential equation

$$
\begin{cases}D_{0^{+}}^{\alpha} x(t)=f\left(t, x(t), x^{\prime}(t), x^{\prime \prime}(t)\right), & t \in[0,1] \\ x(0)=x(1), & x^{\prime}(0)=x^{\prime \prime}(0)=0\end{cases}
$$

where $D_{0^{+}}^{\alpha}$ denotes the Caputo fractional differential operator of order $\alpha, 2<\alpha \leq 3$. A new result on the existence of solutions for above fractional boundary value problem is obtained.

Mathematics Subject Classification (2000): 34A08, 34B15.

Keywords: Fractional differential equations, boundary value problems, resonance, coincidence degree theory

\section{Introduction}

Fractional calculus is a generalization of ordinary differentiation and integration on an arbitrary order that can be noninteger. This subject, as old as the problem of ordinary differential calculus, can go back to the times when Leibniz and Newton invented differential calculus. As is known to all, the problem for fractional derivative was originally raised by Leibniz in a letter, dated September 30, 1695.

In recent years, the fractional differential equations have received more and more attention. The fractional derivative has been occurring in many physical applications such as a non-Markovian diffusion process with memory [1], charge transport in amorphous semiconductors [2], propagations of mechanical waves in viscoelastic media [3], etc. Phenomena in electromagnetics, acoustics, viscoelasticity, electrochemistry, and material science are also described by differential equations of fractional order (see [4-9]).

Recently, boundary value problems (BVPs for short) for fractional differential equations at nonresonance have been studied in many papers (see [10-16]). Moreover, Kosmatov studied the BVPs for fractional differential equations at resonance (see [17]). Motivated by the work above, in this paper, we consider the following BVP of fractional equation at resonance

$$
\begin{cases}D_{0^{+}}^{\alpha} x(t)=f\left(t, x(t), x^{\prime}(t), x^{\prime \prime}(t)\right), & t \in[0,1] \\ x(0)=x(1), & x^{\prime}(0)=x^{\prime \prime}(0)=0\end{cases}
$$

\section{Springer}

(C) 2011 Hu and Liu; licensee Springer. This is an Open Access article distributed under the terms of the Creative Commons Attribution License (http://creativecommons.org/licenses/by/2.0), which permits unrestricted use, distribution, and reproduction in any medium, provided the original work is properly cited. 
where $D_{0^{+}}^{\alpha}$ denotes the Caputo fractional differential operator of order $\alpha, 2<\alpha \leq 3 . f$ $:[0,1] \times \mathbb{R}^{3} \rightarrow \times \mathbb{R}$ is continuous.

The rest of this paper is organized as follows. Section 2 contains some necessary notations, definitions, and lemmas. In Section 3, we establish a theorem on existence of solutions for BVP (1.1) under nonlinear growth restriction of $f$, basing on the coincidence degree theory due to Mawhin (see [18]). Finally, in Section 4, an example is given to illustrate the main result.

\section{Preliminaries}

In this section, we will introduce notations, definitions, and preliminary facts that are used throughout this paper.

Let $X$ and $Y$ be real Banach spaces and let $L: \operatorname{dom} L \subset X \rightarrow Y$ be a Fredholm operator with index zero, and $P: X \rightarrow X, Q: Y \rightarrow Y$ be projectors such that

$$
\begin{aligned}
& \operatorname{Im} P=\operatorname{Ker} L, \quad \operatorname{Ker} Q=\operatorname{Im} L, \\
& X=\operatorname{Ker} L \oplus \operatorname{Ker} P, \quad Y=\operatorname{Im} L \oplus \operatorname{Im} Q .
\end{aligned}
$$

It follows that

$$
\left.L\right|_{\text {domLnKerP }}: \operatorname{dom} L \cap \operatorname{Ker} P \rightarrow \operatorname{Im} L
$$

is invertible. We denote the inverse by $K_{P}$.

If $\Omega$ is an open bounded subset of $X$, and $\operatorname{dom} L \cap \bar{\Omega} \neq \emptyset$, the map $N: X \rightarrow Y$ will be called $L$-compact on $\bar{\Omega}$ if $Q N(\bar{\Omega})$ is bounded and $K_{P}(I-Q) N: \bar{\Omega} \rightarrow X$ is compact. Where $I$ is identity operator.

Lemma 2.1. ([18]) If $\Omega$ is an open bounded set, let $L: \operatorname{dom} L \subset X \rightarrow Y$ be a Fredholm operator of index zero and $N: X \rightarrow Y L$-compact on $\bar{\Omega}$. Assume that the following conditions are satisfied

(1) $L x \neq \lambda N x$ for every $(x, \lambda) \in[(\operatorname{dom} L \backslash \operatorname{Ker} L)] \cap \partial \Omega \times(0,1)$;

(2) $N x \notin \operatorname{Im} L$ for every $x \in \operatorname{Ker} L \cap \partial \Omega$;

(3) $\operatorname{deg}\left(\left.Q N\right|_{\operatorname{Ker} L}, \operatorname{Ker} L \cap \Omega, 0\right) \neq 0$, where $Q: Y \rightarrow Y$ is a projection such that $\operatorname{Im} L=$ KerQ.

Then the equation $L x=N x$ has at least one solution in $\operatorname{dom} L \cap \bar{\Omega}$.

Definition 2.1. The Riemann-Liouville fractional integral operator of order $\alpha>0$ of a function $x$ is given by

$$
I_{0^{+}}^{\alpha} x(t)=\frac{1}{\Gamma(\alpha)} \int_{0}^{t}(t-s)^{\alpha-1} x(s) \mathrm{d} s,
$$

provided that the right side integral is pointwise defined on $(0,+\infty)$.

Definition 2.2. The Caputo fractional derivative of order $\alpha>0$ of a continuous function $x$ is given by

$$
D_{0^{+}}^{\alpha} x(t)=I_{0^{+}}^{n-\alpha} \frac{\mathrm{d}^{n} x(t)}{\mathrm{d} t^{n}}=\frac{1}{\Gamma(n-\alpha)} \int_{0}^{t}(t-s)^{n-\alpha-1} x^{(n)}(s) \mathrm{d} s,
$$

where $n$ is the smallest integer greater than or equal to $\alpha$, provided that the right side integral is pointwise defined on $(0,+\infty)$. 
Lemma 2.2. ([19]) For $\alpha>0$, the general solution of the Caputo fractional differential equation

$$
D_{0^{+}}^{\alpha} x(t)=0
$$

is given by

$$
x(t)=c_{0}+c_{1} t+c_{2} t^{2}+\cdots+c_{n-1} t^{n-1},
$$

where $c_{i} \in \mathbb{R}, i=0,1,2, \ldots, n-1$; here, $\mathrm{n}$ is the smallest integer greater than or equal to $\alpha$.

Lemma 2.3. ([19]) Assume that $x \in C(0,1) \cap L(0,1)$ with a Caputo fractional derivative of order $\alpha>0$ that belongs to $C(0,1) \cap L(0,1)$. Then,

$$
I_{0^{+}}^{\alpha} D_{0^{+}}^{\alpha} x(t)=x(t)+c_{0}+c_{1} t+c_{2} t^{2}+\cdots+c_{n-1} t^{n-1}
$$

where $c_{i} \in \mathbb{R}, i=0,1,2, \ldots, n-1$; here, $\mathrm{n}$ is the smallest integer greater than or equal to $\alpha$.

In this paper, we denote $X=C^{2}[0,1]$ with the norm $\|x\|_{X}=\max \left\{\|x\|_{\infty},\left\|x^{\prime}\right\|_{\infty}, \|\right.$ $\left.x^{\prime \prime} \mid \|_{\infty}\right\}$ and $Y=C[0,1]$ with the norm $\|y\|_{Y}=\|y\|_{\infty}$, where $\|x\|_{\infty}=\max _{t \in[0,1]}|x(t)|$. Obviously, both $X$ and $Y$ are Banach spaces.

Define the operator $L: \operatorname{dom} L \subset X \rightarrow Y$ by

$$
L x=D_{0^{+}}^{\alpha} x,
$$

where

$$
\operatorname{dom} L=\left\{x \in X \mid D_{0^{+}}^{\alpha} x(t) \in Y, x(0)=x(1), x^{\prime}(0)=x^{\prime \prime}(0)=0\right\} .
$$

Let $N: X \rightarrow Y$ be the Nemytski operator

$$
N x(t)=f\left(t, x(t), x^{\prime}(t), x^{\prime \prime}(t)\right), \quad \forall t \in[0,1] .
$$

Then, BVP (1.1) is equivalent to the operator equation

$$
L x=N x, \quad x \in \operatorname{dom} L .
$$

\section{Main result}

In this section, a theorem on existence of solutions for BVP (1.1) will be given.

Theorem 3.1. Let $f:[0,1] \times \mathbb{R}^{3} \rightarrow \mathbb{R}$ be continuous. Assume that

$\left(H_{1}\right)$ there exist nonnegative functions $p, q, r, s \in C[0,1]$ with $\Gamma(\alpha-1)-q_{1}-r_{1}-s_{1}>$ 0 such that

$$
|f(t, u, v, w)| \leq p(t)+q(t)|u|+r(t)|v|+s(t)|w|, \quad \forall t \in[0,1], \quad(u, v, w) \in \mathbb{R}^{3},
$$

where $p_{1}=\|p\|_{\infty}, q_{1}=\|q\|_{\infty}, r_{1}=\|r\|_{\infty}, s_{1}=\|s\|_{\infty}$.

$\left(H_{2}\right)$ there exists a constant $B>0$ such that for all $u \in \mathbb{R}$ with $|u|>B$ either

$$
u f(t, u, v, w)>0, \quad \forall t \in[0,1],(v, w) \in \mathbb{R}^{2}
$$

or

$$
u f(t, u, v, w)<0, \quad \forall t \in[0,1],(v, w) \in \mathbb{R}^{2} .
$$


Then, BVP (1.1) has at leat one solution in $X$.

Now, we begin with some lemmas below.

Lemma 3.1. Let $L$ be defined by (2.1), then

$$
\begin{aligned}
& \operatorname{Ker} L=\left\{x \in X \mid x(t)=c_{0}, c_{0} \in \mathbb{R}, \forall t \in[0,1]\right\} \\
& \operatorname{Im} L=\left\{y \in Y \mid \int_{0}^{1}(1-s)^{\alpha-1} \gamma(s) \mathrm{d} s=0\right\}
\end{aligned}
$$

Proof. By Lemma 2.2, $D_{0^{+}}^{\alpha} x(t)=0$ has solution

$$
x(t)=c_{0}+c_{1} t+c_{2} t^{2}, \quad c_{0}, c_{1}, c_{2} \in \mathbb{R} .
$$

Combining with the boundary value condition of BVP (1.1), one has (3.1) hold.

For $y \in \operatorname{Im} L$, there exists $x \in \operatorname{dom} L$ such that $y=L x \in Y$. By Lemma 2.3, we have

$$
x(t)=\frac{1}{\Gamma(\alpha)} \int_{0}^{t}(t-s)^{\alpha-1} \gamma(s) \mathrm{d} s+c_{0}+c_{1} t+c_{2} t^{2} .
$$

Then, we have

$$
x^{\prime}(t)=\frac{1}{\Gamma(\alpha-1)} \int_{0}^{t}(t-s)^{\alpha-2} \gamma(s) \mathrm{d} s+c_{1}+2 c_{2} t
$$

and

$$
x^{\prime \prime}(t)=\frac{1}{\Gamma(\alpha-2)} \int_{0}^{t}(t-s)^{\alpha-3} \gamma(s) \mathrm{d} s+2 c_{2} .
$$

By conditions of BVP (1.1), we can get that $y$ satisfies

$$
\int_{0}^{1}(1-s)^{\alpha-1} y(s) \mathrm{d} s=0 .
$$

Thus, we get (3.2). On the other hand, suppose $y \in Y$ and satisfies $\int_{0}^{1}(1-s)^{\alpha-1} \gamma(s) \mathrm{d} s=0$. Let $x(t)=I_{0^{+}}^{\alpha} y(t)$, then $x \in \operatorname{dom} L$ and $D_{0^{+}}^{\alpha} x(t)=\gamma(t)$. So that, $y \in \operatorname{Im} L$. The proof is complete.

Lemma 3.2. Let $L$ be defined by (2.1), then $L$ is a Fredholm operator of index zero, and the linear continuous projector operators $P: X \rightarrow X$ and $Q: Y \rightarrow Y$ can be defined as

$$
\begin{aligned}
& P x(t)=x(0), \quad \forall t \in[0,1] \\
& Q y(t)=\alpha \int_{0}^{1}(1-s)^{\alpha-1} y(s) \mathrm{d} s, \quad \forall t \in[0,1] .
\end{aligned}
$$


Furthermore, the operator $K_{P}: \operatorname{Im} L \rightarrow \operatorname{dom} L \cap \operatorname{Ker} P$ can be written by

$$
K_{P} y(t)=\frac{1}{\Gamma(\alpha)} \int_{0}^{t}(t-s)^{\alpha-1} \gamma(s) \mathrm{d} s, \quad \forall t \in[0,1] .
$$

Proof. Obviously, $\operatorname{Im} P=\operatorname{Ker} L$ and $P^{2} x=P x$. It follows from $x=(x-P x)+P x$ that $X$ $=\operatorname{Ker} P+\operatorname{Ker} L$. By simple calculation, we can get that $\operatorname{Ker} L \cap \operatorname{Ker} P=\{0\}$. Then, we get

$$
X=\operatorname{Ker} L \oplus \operatorname{Ker} P .
$$

For $y \in Y$, we have

$$
Q^{2} y=Q(Q y)=Q y \cdot \alpha \int_{0}^{1}(1-s)^{\alpha-1} \mathrm{~d} s=Q y .
$$

Let $y=(y-Q y)+Q y$, where $y-Q y \in \operatorname{Ker} Q=\operatorname{Im} L, Q y \in \operatorname{Im} Q$. It follows from KerQ $=\operatorname{Im} L$ and $Q^{2} y=Q y$ that $\operatorname{Im} Q \cap \operatorname{Im} L=\{0\}$. Then, we have

$$
Y=\operatorname{Im} L \oplus \operatorname{Im} Q .
$$

Thus,

$$
\operatorname{dim} \operatorname{Ker} L=\operatorname{dim} \operatorname{Im} Q=\operatorname{codim} \operatorname{Im} L=1 .
$$

This means that $L$ is a Fredholm operator of index zero.

From the definitions of $P, K_{P}$, it is easy to see that the generalized inverse of $L$ is $K_{P}$. In fact, for $y \in \operatorname{Im} L$, we have

$$
L K_{P} y=D_{0^{+}}^{\alpha} I_{0^{+}}^{\alpha} y=y .
$$

Moreover, for $x \in \operatorname{dom} L \cap \operatorname{Ker} P$, we get $x(0)=x^{\prime}(0)=x^{\prime \prime}(0)=0$. By Lemma 2.3, we obtain that

$$
I_{0^{+}}^{\alpha} L x(t)=I_{0^{+}}^{\alpha} D_{0^{+}}^{\alpha} x(t)=x(t)+c_{0}+c_{1} t+c_{2} t^{2}, \quad c_{0}, c_{1}, c_{2} \in \mathbb{R},
$$

which together with $x(0)=x^{\prime}(0)=x^{\prime \prime}(0)=0$ yields that

$$
K_{P} L x=x .
$$

Combining (3.3) with (3.4), we know that $K_{P}=\left(\left.L\right|_{\operatorname{dom} L \cap K e r P}\right)^{-1}$. The proof is complete.

Lemma 3.3. Assume $\Omega \subset X$ is an open bounded subset such that $\operatorname{dom} L \cap \bar{\Omega} \neq \emptyset$, then $N$ is $L$-compact on $\bar{\Omega}$.

Proof. By the continuity of $f$, we can get that $Q N(\bar{\Omega})$ and $K_{P}(I-Q) N(\bar{\Omega})$ are bounded. So, in view of the Arzelà -Ascoli theorem, we need only prove that $K_{P}(I-Q) N(\bar{\Omega}) \subset X$ is equicontinuous.

From the continuity of $f$, there exists constant $A>0$ such that $|(I-Q) N x| \leq A$, $\forall x \in \bar{\Omega}, t \in[0,1]$. Furthermore, denote $K_{P, Q}=K_{P}(I-Q) N$ and for $0 \leq t_{1}<t_{2} \leq 1$, $x \in \bar{\Omega}$, we have 


$$
\begin{aligned}
& \left|\left(K_{P, Q} x\right)\left(t_{2}\right)-\left(K_{P, Q} x\right)\left(t_{1}\right)\right| \\
& \quad \leq \frac{1}{\Gamma(\alpha)}\left|\int_{0}^{t_{2}}\left(t_{2}-s\right)^{\alpha-1}(I-Q) N x(s) \mathrm{d} s-\int_{0}^{t_{1}}\left(t_{1}-s\right)^{\alpha-1}(I-Q) N x(s) \mathrm{d} s\right| \\
& \quad \leq \frac{A}{\Gamma(\alpha)}\left[\int_{0}^{t_{1}}\left(t_{2}-s\right)^{\alpha-1}-\left(t_{1}-s\right)^{\alpha-1} \mathrm{~d} s+\int_{t_{1}}^{t_{2}}\left(t_{2}-s\right)^{\alpha-1} \mathrm{~d} s\right] \\
& \quad=\frac{A}{\Gamma(\alpha+1)}\left(t_{2}^{\alpha}-t_{1}^{\alpha}\right), \\
& \left|\left(K_{P, Q} x\right)^{\prime}\left(t_{2}\right)-\left(K_{P, Q} x\right)^{\prime}\left(t_{1}\right)\right| \\
& \quad=\frac{\alpha-1}{\Gamma(\alpha)}\left|\int_{0}^{t_{2}}\left(t_{2}-s\right)^{\alpha-2}(I-Q) N x(s) \mathrm{d} s-\int_{0}^{t_{1}}\left(t_{1}-s\right)^{\alpha-2}(I-Q) N x(s) \mathrm{d} s\right| \\
& \quad \leq \frac{A}{\Gamma(\alpha-1)}\left[\int_{0}^{t_{1}}\left(t_{2}-s\right)^{\alpha-2}-\left(t_{1}-s\right)^{\alpha-2} \mathrm{~d} s+\int_{t_{1}}^{t_{2}}\left(t_{2}-s\right)^{\alpha-2} \mathrm{~d} s\right] \\
& \quad \leq \frac{A}{\Gamma(\alpha)}\left(t_{2}^{\alpha-1}-t_{1}^{\alpha-1}\right)
\end{aligned}
$$

and

$$
\begin{aligned}
& \left|\left(K_{P, Q} x\right)^{\prime \prime}\left(t_{2}\right)-\left(K_{P, Q} x\right)^{\prime \prime}\left(t_{1}\right)\right| \\
& \quad=\frac{(\alpha-2)(\alpha-1)}{\Gamma(\alpha)}\left|\int_{0}^{t_{2}}\left(t_{2}-s\right)^{\alpha-3}(I-Q) N x(s) \mathrm{d} s-\int_{0}^{t_{1}}\left(t_{1}-s\right)^{\alpha-3}(I-Q) N x(s) \mathrm{d} s\right| \\
& \quad \leq \frac{A}{\Gamma(\alpha-2)}\left[\int_{0}^{t_{1}}\left(t_{1}-s\right)^{\alpha-3}-\left(t_{2}-s\right)^{\alpha-3} \mathrm{~d} s+\int_{t_{1}}^{t_{2}}\left(t_{2}-s\right)^{\alpha-3} \mathrm{~d} s\right] \\
& \quad \leq \frac{A}{\Gamma(\alpha-1)}\left[t_{1}^{\alpha-2}-t_{2}^{\alpha-2}+2\left(t_{2}-t_{1}\right)^{\alpha-2}\right] .
\end{aligned}
$$

Since $t^{\alpha}, t^{\alpha-1}$ and $t^{\alpha-2}$ are uniformly continuous on $[0,1]$, we can get that $\left(K_{P, Q}\right)^{\prime}(\bar{\Omega}) \subset C[0,1],\left(K_{P, Q}\right)^{\prime}(\bar{\Omega}) \subset C[0,1]$ and $\left(K_{P, Q}\right)^{\prime \prime}(\bar{\Omega}) \subset C[0,1]$ are equicontinuous. Thus, we get that $K_{P, Q}: \bar{\Omega} \rightarrow X$ is compact. The proof is completed.

Lemma 3.4. Suppose $\left(H_{1}\right),\left(H_{2}\right)$ hold, then the set

$$
\Omega_{1}=\{x \in \operatorname{dom} L \backslash \operatorname{Ker} L \mid L x=\lambda N x, \quad \lambda \in(0,1)\}
$$

is bounded.

Proof. Take $x \in \Omega_{1}$, then $N x \in \operatorname{Im} L$. By (3.2), we have

$$
\int_{0}^{1}(1-s)^{\alpha-1} f\left(s, x(s), x^{\prime}(s), x^{\prime \prime}(s)\right) \mathrm{d} s=0 .
$$

Then, by the integral mean value theorem, there exists a constant $\xi \in(0,1)$ such that $f\left(\xi, x(\xi), x^{\prime}(\xi), x^{\prime \prime}(\xi)\right)=0$. Then from $\left(H_{2}\right)$, we have $|x(\xi)| \leq B$. 
Then, we have

$$
|x(t)|=\left|x(\xi)+\int_{\xi}^{t} x^{\prime}(s) \mathrm{d} s\right| \leq B+\left\|x^{\prime}\right\|_{\infty} .
$$

That is

$$
\|x\|_{\infty} \leq B+\left\|x^{\prime}\right\|_{\infty} .
$$

From $x \in \operatorname{dom} L$, we get $x^{\prime}(0)=0$. Therefore,

$$
\left|x^{\prime}(t)\right|=\left|x^{\prime}(0)+\int_{0}^{t} x^{\prime \prime}(s) \mathrm{d} s\right| \leq\left\|x^{\prime \prime}\right\|_{\infty}
$$

That is

$$
\left\|x^{\prime}\right\|_{\infty} \leq\left\|x^{\prime \prime}\right\|_{\infty}
$$

By $L x=\lambda N x$ and $x \in \operatorname{dom} L$, we have

$$
x(t)=\frac{\lambda}{\Gamma(\alpha)} \int_{0}^{t}(t-s)^{\alpha-1} f\left(s, x(s), x^{\prime}(s), x^{\prime \prime}(s)\right) \mathrm{d} s+x(0) .
$$

Then we get

$$
x^{\prime}(t)=\frac{\lambda}{\Gamma(\alpha-1)} \int_{0}^{t}(t-s)^{\alpha-2} f\left(s, x(s), x^{\prime}(s), x^{\prime \prime}(s)\right) \mathrm{d} s
$$

and

$$
x^{\prime \prime}(t)=\frac{\lambda}{\Gamma(\alpha-2)} \int_{0}^{t}(t-s)^{\alpha-3} f\left(s, x(s), x^{\prime}(s), x^{\prime \prime}(s)\right) \mathrm{d} s .
$$

From (3.5),(3.6), and $\left(H_{1}\right)$, we have

$$
\begin{aligned}
\left\|x^{\prime \prime}\right\|_{\infty} & \leq \frac{1}{\Gamma(\alpha-2)} \int_{0}^{t}(t-s)^{\alpha-3}\left|f\left(s, x(s), x^{\prime}(s), x^{\prime \prime}(s)\right)\right| \mathrm{d} s \\
& \leq \frac{1}{\Gamma(\alpha-2)} \int_{0}^{t}(t-s)^{\alpha-3}\left[p(s)+q(s)|x(s)|+r(s)\left|x^{\prime}(s)\right|+s(s)\left|x^{\prime \prime}(s)\right|\right] \mathrm{d} s \\
& \leq \frac{1}{\Gamma(\alpha-2)} \int_{0}^{t}(t-s)^{\alpha-3}\left(p_{1}+q_{1}\|x\|_{\infty}+r_{1}\left\|x^{\prime}\right\|_{\infty}+s_{1}\left\|x^{\prime \prime}\right\|_{\infty}\right) \mathrm{d} s \\
& \leq \frac{1}{\Gamma(\alpha-2)} \int_{0}^{t}(t-s)^{\alpha-3}\left[p_{1}+q_{1} B+\left(q_{1}+r_{1}+s_{1}\right)\left\|x^{\prime \prime}\right\|_{\infty}\right] \mathrm{d} s \\
& \leq \frac{1}{\Gamma(\alpha-1)}\left[p_{1}+q_{1} B+\left(q_{1}+r_{1}+s_{1}\right)\left\|x^{\prime \prime}\right\|_{\infty}\right] .
\end{aligned}
$$


Thus, from $\Gamma(\alpha-1)-q_{1}-r_{1}-s_{1}>0$, we obtain that

$$
\left\|x^{\prime \prime}\right\|_{\infty} \leq \frac{p_{1}+q_{1} B}{\Gamma(\alpha-1)-q_{1}-r_{1}-s_{1}}:=M_{1} .
$$

Thus, we get

$$
\left\|x^{\prime}\right\|_{\infty} \leq\left\|x^{\prime \prime}\right\|_{\infty} \leq M_{1}
$$

and

$$
\|x\|_{\infty} \leq B+\left\|x^{\prime}\right\|_{\infty} \leq B+M_{1} .
$$

Therefore,

$$
\|x\|_{X} \leq \max \left\{M_{1}, B+M_{1}\right\} .
$$

So $\Omega_{1}$ is bounded. The proof is complete.

Lemma 3.5. Suppose $\left(H_{2}\right)$ holds, then the set

$$
\Omega_{2}=\{x \mid x \in \operatorname{Ker} L, N x \in \operatorname{Im} L\}
$$

is bounded.

Proof. For $x \in \Omega_{2}$, we have $x(t)=c, c \in \mathbb{R}$, and $N x \in \operatorname{Im} L$. Then, we get

$$
\int_{0}^{1}(1-s)^{\alpha-1} f(s, c, 0,0) \mathrm{d} s=0
$$

which together with $\left(H_{2}\right)$ implies $|c| \leq B$. Thus, we have

$$
\|x\|_{X} \leq B
$$

Hence, $\Omega_{2}$ is bounded. The proof is complete.

Lemma 3.6. Suppose the first part of $\left(H_{2}\right)$ holds, then the set

$$
\Omega_{3}=\{x \mid x \in \operatorname{Ker} L, \lambda x+(1-\lambda) Q N x=0, \quad \lambda \in[0,1]\}
$$

is bounded.

Proof. For $x \in \Omega_{3}$, we have $x(t)=c, c \in \mathbb{R}$, and

$$
\lambda c+(1-\lambda) \alpha \int_{0}^{1}(1-s)^{\alpha-1} f(s, c, 0,0) \mathrm{d} s=0 .
$$

If $\lambda=0$, then $|c| \leq B$ because of the first part of $\left(H_{2}\right)$. If $\lambda \in(0,1]$, we can also obtain $|c| \leq B$. Otherwise, if $|c|>B$, in view of the first part of $\left(H_{2}\right)$, one has

$$
\lambda c^{2}+(1-\lambda) \alpha \int_{0}^{1}(1-s)^{\alpha-1} c f(s, c, 0,0) \mathrm{d} s>0,
$$

which contradicts to (3.7).

Therefore, $\Omega_{3}$ is bounded. The proof is complete. 
Remark 3.1. Suppose the second part of $\left(H_{2}\right)$ hold, then the set

$$
\Omega_{3}^{\prime}=\{x \mid x \in \operatorname{Ker} L,-\lambda x+(1-\lambda) Q N x=0, \quad \lambda \in[0,1]\}
$$

is bounded.

The proof of Theorem 3.1. Set $\Omega=\left\{x \in X \mid\|x\|_{X}<\max \left\{M_{1}, B, B+M_{1}\right\}+1\right\}$. It follows from Lemma 3.2 and 3.3 that $L$ is a Fredholm operator of index zero and $N$ is $L$-compact on $\bar{\Omega}$. By Lemma 3.4 and 3.5, we get that the following two conditions are satisfied

(1) $L x \neq \lambda N x$ for every $(x, \lambda) \in[(\operatorname{dom} L \backslash \operatorname{Ker} L) \cap \partial \Omega] \times(0,1)$;

(2) $N x \notin \operatorname{Im} L$ for every $x \in \operatorname{Ker} L \cap \partial \Omega$.

Take

$$
H(x, \lambda)= \pm \lambda x+(1-\lambda) Q N x .
$$

According to Lemma 3.6 (or Remark 3.1), we know that $H(x, \lambda) \neq 0$ for $x \in \operatorname{Ker} L \cap$ $\partial \Omega$. Therefore,

$$
\begin{aligned}
\operatorname{deg}\left(\left.Q N\right|_{\text {KerL }}, \Omega \cap \operatorname{KerL}, 0\right) & =\operatorname{deg}(H(\cdot, 0), \Omega \cap \operatorname{Ker} L, 0) \\
& =\operatorname{deg}(H(\cdot, 1), \Omega \cap \operatorname{Ker} L, 0) \\
& =\operatorname{deg}( \pm I, \Omega \cap \operatorname{Ker} L, 0) \neq 0 .
\end{aligned}
$$

So that, the condition (3) of Lemma 2.1 is satisfied. By Lemma 2.1, we can get that $L x=N x$ has at least one solution in $\operatorname{dom} L \cap \bar{\Omega}$. Therefore, BVP (1.1) has at least one solution. The proof is complete.

\section{An example}

Example 4.1. Consider the following BVP

$$
\begin{cases}D_{0^{+}}^{\frac{5}{2}} x(t)=\frac{t}{16}(x-10)+\frac{t^{2}}{16} e^{-\left|x^{\prime}\right|}+\frac{t^{3}}{16} \sin \left[\left(x^{\prime \prime}\right)^{2}\right], & t \in[0,1] \\ x(0)=x(1), & x^{\prime}(0)=x^{\prime \prime}(0)=0 .\end{cases}
$$

where

$$
f(t, u, v, w)=\frac{t}{16}(u-10)+\frac{t^{2}}{16} e^{-|v|}+\frac{t^{3}}{16} \sin \left(w^{2}\right) .
$$

Choose $p(t)=\frac{10 t+2}{16}, q(t)=\frac{t}{16}, r(t)=0, s(t)=0, B=10$. We can get that $q_{1}=\frac{1}{16}, r_{1}$ $=0, s_{1}=0$ and

$$
\Gamma\left(\frac{5}{2}-1\right)-q_{1}-r_{1}-s_{1}>0 .
$$

Then, all conditions of Theorem 3.1 hold, so BVP (4.1) has at least one solution.

\section{Acknowledgements}

The authors would like to thank the referees very much for their helpful comments and suggestions. This research was supported by the Fundamental Research Funds for the Central Universities (2010LKSX09) and the Science Foundation of China University of Mining and Technology (2008A037). 


\section{Competing interests}

The authors declare that they have no competing interests.

Received: 10 May 2011 Accepted: 5 September 2011 Published: 5 September 2011

\section{References}

1. Metzler, R, Klafter, J: Boundary value problems for fractional diffusion equations. Phys A. 278, 107-125 (2000). doi:10.1016/S0378-4371(99)00503-8

2. Scher, H, Montroll, E: Anomalous transit-time dispersion in amorphous solids. Phys Rev B. 12, 2455-2477 (1975). doi:10.1103/PhysRevB.12.2455

3. Mainardi, F: Fractional diffusive waves in viscoelastic solids. In: Wegner JL, Norwood FR (eds.) Nonlinear Waves in Solids. pp. 93-97. ASME/AMR, Fairfield NJ (1995)

4. Diethelm, K, Freed, AD: On the solution of nonlinear fractional order differential equations used in the modeling of viscoplasticity. In: Keil F, Mackens W, Voss H, Werther J (eds.) Scientific Computing in Chemical Engineering IIComputational Fluid Dynamics, Reaction Engineering and Molecular Properties. pp. 217-224. Springer, Heidelberg (1999)

5. Gaul, L, Klein, P, Kempfle, S: Damping description involving fractional operators. Mech Syst Signal Process. 5, 81-88 (1991). doi:10.1016/0888-3270(91)90016-X

6. Glockle, WG, Nonnenmacher, TF: A fractional calculus approach of self-similar protein dynamics. Biophys J. 68, 46-53 (1995). doi:10.1016/50006-3495(95)80157-8

7. Mainardi, F: Fractional calculus: some basic problems in continuum and statistical mechanics. In: Carpinteri A, Mainardi F (eds.) Fractals and Fractional Calculus in Continuum Mechanics. pp. 291-348. Springer, Wien (1997)

8. Metzler, F, Schick, W, Kilian, HG, Nonnenmacher, TF: Relaxation in filled polymers: a fractional calculus approach. J Chem Phys. 103, 7180-7186 (1995). doi:10.1063/1.470346

9. Oldham, KB, Spanier, J: The Fractional Calculus. Academic Press, New York, London (1974)

10. Agarwal, RP, ORegan, D, Stanek, S: Positive solutions for Dirichlet problems of singular nonlinear fractional differential equations. J Math Anal Appl. 371, , 57-68 (2010)

11. Bai, Z, Hu, L: Positive solutions for boundary value problem of nonlinear fractional differential equation. J Math Anal Appl. 311, 495-505 (2005). doi:10.1016/j.jmaa.2005.02.052

12. Kaufmann, ER, Mboumi, E: Positive solutions of a boundary value problem for a nonlinear fractional differential equation. Electron J Qual Theory Differ Equ. 3, 1-11 (2008)

13. Jafari, H, Gejji, VD: Positive solutions of nonlinear fractional boundary value problems using Adomian decomposition method. Appl Math Comput. 180, 700-706 (2006). doi:10.1016/.amc.2006.01.007

14. Benchohra, M, Hamani, S, Ntouyas, SK: Boundary value problems for differential equations with fractional order and nonlocal conditions. Nonlinear Anal. 71, 2391-2396 (2009). doi:10.1016/j.na.2009.01.073

15. Liang, S, Zhang, J: Positive solutions for boundary value problems of nonlinear fractional differential equation. Nonlinear Anal. 71, 5545-5550 (2009). doi:10.1016/..na.2009.04.045

16. Zhang, S: Positive solutions for boundary-value problems of nonlinear fractional differential equations. Electron J Differ Equ. 36, 1-12 (2006)

17. Kosmatov, N: A boundary value problem of fractional order at resonance. Electron J Differ Equ. 135, 1-10 (2010)

18. Mawhin, J: Topological degree and boundary value problems for nonlinear differential equations in topological methods for ordinary differential equations. Lect Notes Math. 1537, 74-142 (1993). doi:10.1007/BFb0085076

19. Lakshmikantham, V, Leela, S, Vasundhara Devi, J: Theory of Fractional Dynamic Systems. Cambridge Academic Publishers, Cambridge (2009)

doi:10.1186/1687-2770-2011-20

Cite this article as: $\mathrm{Hu}$ and Liu: Solvability for fractional order boundary value problems at resonance. Boundary Value Problems 2011 2011:20.

\section{Submit your manuscript to a SpringerOpen ${ }^{\odot}$ journal and benefit from:}

- Convenient online submission

Rigorous peer review

- Immediate publication on acceptance

- Open access: articles freely available online

- High visibility within the field

- Retaining the copyright to your article

Submit your next manuscript at $\boldsymbol{\wedge}$ springeropen.com 